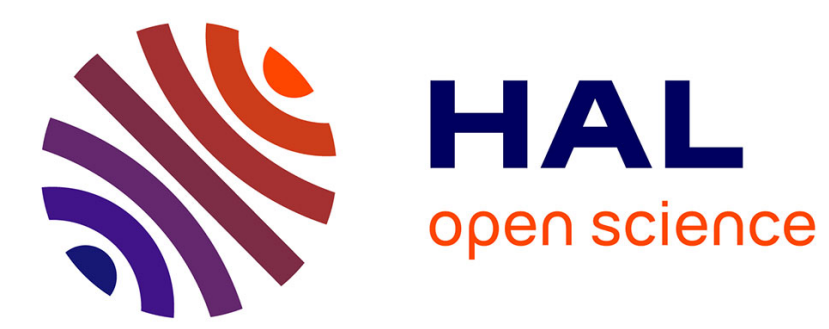

\title{
Progress in direct noise computation
}

Christophe Bailly, Christophe Bogey, Olivier Marsden

\section{To cite this version:}

Christophe Bailly, Christophe Bogey, Olivier Marsden. Progress in direct noise computation. International Journal of Aeroacoustics, 2010, 9, pp.123-143. 10.1260/1475-472X.9.1-2.123 . hal-00566027

\section{HAL Id: hal-00566027 \\ https://hal.science/hal-00566027}

Submitted on 8 Jun 2012

HAL is a multi-disciplinary open access archive for the deposit and dissemination of scientific research documents, whether they are published or not. The documents may come from teaching and research institutions in France or abroad, or from public or private research centers.
L'archive ouverte pluridisciplinaire HAL, est destinée au dépôt et à la diffusion de documents scientifiques de niveau recherche, publiés ou non, émanant des établissements d'enseignement et de recherche français ou étrangers, des laboratoires publics ou privés. 


\title{
Progress in direct noise computation
}

\author{
Christophe Bailly*, Christophe Bogey, and Olivier Marsden \\ Laboratoire de Mécanique des Fluides et d'Acoustique, \\ Ecole Centrale de Lyon \& CNRS UMR CNRS 5509 \\ 36 avenue Guy de Collongue, 69134 Ecully cedex, France
}

Received October 13, 2008; Revised April 15, 2009; Accepted for publication April 27, 2009

\begin{abstract}
The needs for accurate and efficient numerical solvers in computational aeroacoustics have motivated the development of low-dispersion and low-dissipation schemes as an alternative to more classical methods of applied mathematics for computational fluid mechanics over the last two decades. These numerical methods have now reached maturity, even if progress is still necessary to take account of specific physics. The paper provides a short overview of some recent developments and applications involving the direct computation of aerodynamic noise with applications to subsonic and supersonic jet noise, to cavity noise and to self-excited internal flows.
\end{abstract}

\section{INTRODUCTION}

Direct Noise Computation (DNC) consists in solving the compressible Navier-Stokes equations to determine simultaneously the aerodynamic field and the acoustic field in a same domain. This approach is quite different from more classical modellings for which aerodynamics and acoustics are decoupled, such as Lighthill's analogy [26]. It is consequently rather natural to apply this approach for studying in more detail noise mechanisms and modelling, and for evaluating noise reduction solutions. The resolution of more theoretical problems concerning aeroacoustics and propagation in the presence of a flow can also be performed by this way. Note that excellent technical reviews on computational aeroacoustics are available, for instance, Colonius and Lele [16], Wang, Freund \& Lele [50] or Colonius [15] for the key problem of non-reflecting boundary conditions. In the present contribution, we focus on the use of DNC for various problems including free shear flows, confined flows and coupling with acoustic resonance. The spectacular development of computational aeroacoustics since the beginning of the nineties has allowed the emergence of the direct computation of

\footnotetext{
* Corresponding author: Christophe Bailly, christophe.bailly@ec-lyon.fr
} 


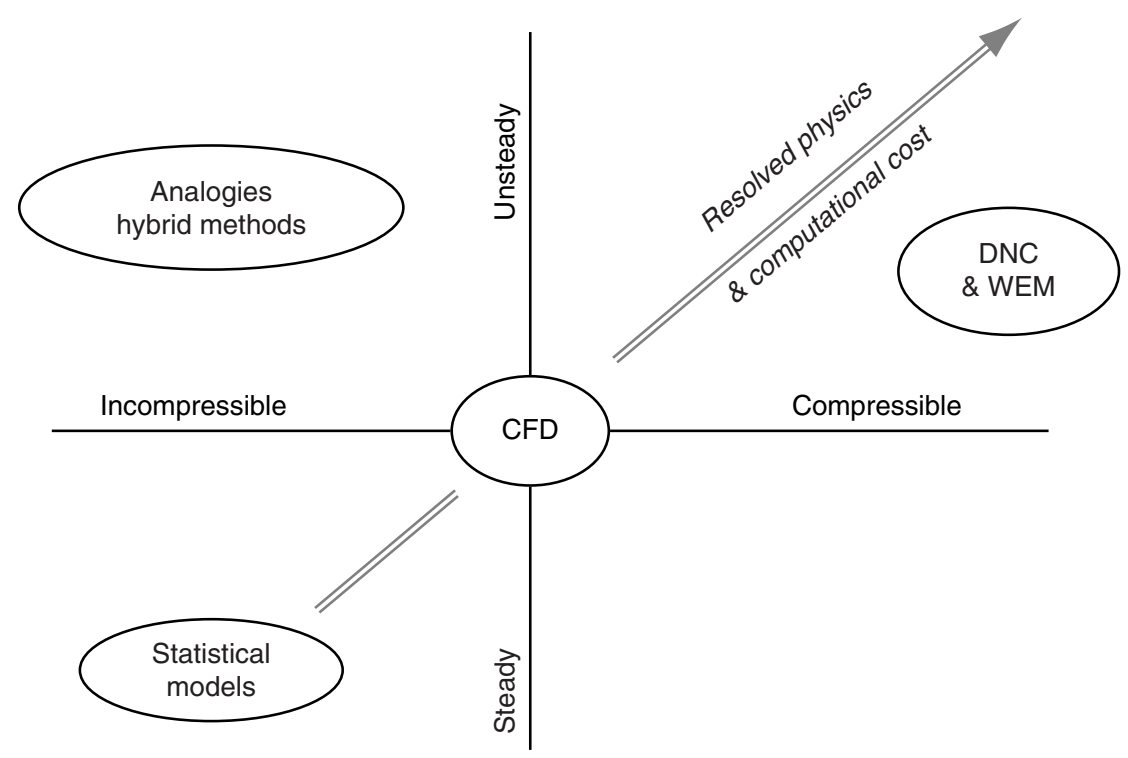

Figure 1: Different modelling levels in aeroacoustics with respect to Conputational Fluid Dynamics (CFD) simulations. DNC stands for Direct Noise Computation and WEM for Wave Extrapolation Methods.

aerodynamic noise, which is now technically mature. There is still a lot of scope for progress, in particular for the numerics and for the strategies to implement for more complex configurations. But direct noise calculation is currently a reliable and accurate tool, which reproduces studied physics with high fidelity.

From a more general point of view however, and to mention another important domain of application, DNC cannot be the classical procedure for low Mach number flows often encountered in automotive applications for instance. DNC would involve heavy computations whereas more suitable and efficient approaches are also possible, see the sketch in figure 1. In this classification, statistical or algebraic models require only the turbulent mean flow field as input data [2,3,33]. These approaches are thus simple but they include a limited description of physical interactions. A stochastic turbulent field can also be reconstructed to compute time-dependent aeroacoustic source terms, as the Stochastic Noise Generation and Radiation (SNGR) model $[4,35]$. Wave extrapolation methods are the natural way to reach the radiated far field from a DNC including only a small part of the acoustic region. The integral formulation of Ffowcs Williams \& Hawkings [20], the linearized Euler equations [4] or the use of a convected wave-operator [12] are included in this second category of methods. Finally, acoustic analogies or hybrid formulations have been developed in computational aeroacoustics, with respect to the compressible and unsteady features of CFD simulations, and also with the aim to take account for mean flow - sound 
(a)

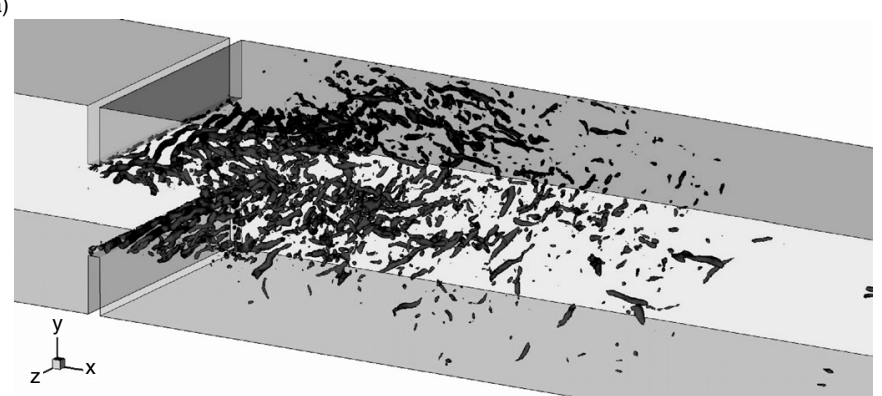

(b)

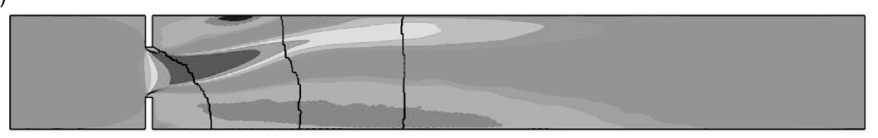

Figure 2: $\quad$ Turbulent flow inside a 3-D diaphragm computed by LES, see Piellard et al. [36] for more details. (a) snaphot of Q-criterium isosurfaces are plotted for the value $Q \times\left(h / u_{\infty}\right)^{2}=10$, and (b) averaged mean axial velocity along the $x-y$ center plane. $Q$ is defined as $Q=\left(\Omega_{i j} \Omega_{i j}-S_{i j} S_{i j}\right) / 2$ where $\Omega_{i j}$ and $S_{i j}$ are the antisymmetric and the symmetric parts of the velocity gradient tensor. The bulk velocity is $u_{\infty}=20 \mathrm{~m} \cdot \mathrm{s}^{-1}$ and the inlet channel height is $h=0.035 \mathrm{~m}$ yielding a Reynolds number $\operatorname{Re}_{h}=4.7 \times 10^{4}$. The expansion ratio is $H / h \simeq 2.3$, the aspect ratio is $w / h \simeq 2.9$ and the length of the main channel is $L / h \simeq 14.3$.

waves interactions [4,24,32,38]. For low-Mach number flows, convection and refractions effects are not an issue, noise generation and sound propagation are often clearly separated, which fully justifies the use of an analogy. Aerodynamic sources are indeed compact and the characteristic scale of the source region and of the radiated acoustic field are therefore disparate.

As an illustration of this point, noise emitted by HVAC - heating, ventilating and air conditioning - is considered with the turbulent flow through a three-dimensional diaphragm used as a nominally representative configuration [36]. Snaphots of the Q-criterium and the mean axial velocity along the $x-y$ center plane are shown in figure 2 . The mean flow is asymmetric behind the sudden expansion and is deviated, as expected by comparison with measurements, towards the upper side wall in the present case. Noise may then be computed in a second step by using a variational formulation of Lighthill's analogy in Fourier space proposed by Oberai et al. [34] as implemented in some commercial codes. Such an approach requires the correct description of spatial source terms, typically of the form $\partial\left(\rho u_{i} u_{j}\right) / \partial x_{j}$ and a windowing of the source volume to control the truncation. These topics are well known in computational aeroacoustics, and in particular, the application of 
a formulation based on space derivatives does not provide the best accuracy at a given mesh. The acoustic step is difficult to perform and finally requires prohibitive computing ressources for fair acoustic predictions. One alternative is to apply the surface integral formulation of Curle [17] for instance, by noting however that the simulation of the turbulent flow is incompressible [22]. These remarks underline some difficulties for coupling a CFD code with a CAA code for noise predictions, and more generally, for assessing modelling levels in aeroacoustics in real life.

New developments in DNC are still being made in all the fields of computational aeroacoustics, and the objectives of this paper are to underline some of them by keeping a general point of view. The text is organized as follows. The constant progress of numerics is outlined in section 2 by the presentation of an optimized low-storage 4thorder Runge-Kutta scheme for which the dissipation error is spectacularly reduced. Section 3 is devoted to noise radiated by round subsonic jets, and thus to broadband noise associated with high-Reynolds-number turbulent free shear flows. Analysis of noise sources by a causality method is illustrated. In sections 4 and 5, noise radiated by a planar imperfectly expanded supersonic jet and by self-induced supersonic flow oscillations behind a sudden enlargement are discussed. In these two examples, the presence of a feedback mechanism and/or of resonances for internal flows often introduces a frequency selection. The involved scales, e.g. scales associated with wall flows, shocks and acoustic resonances in duct flows, are also strongly disparate. In section 6, the simulation of an adaptive control to reduce cavity noise is presented. Work in progress is finally mentioned.

\section{NUMERICS}

The algorithms used in the direct noise computation require a continuous effort of development to improve numerical efficiency, allowing the simulation of more complex configurations including physics and geometry. As an example of recent development which could significantly improve numerical simulations, the optimized Runge-Kutta scheme developed by Berland et al. [5] is now discussed. This point illustrates the effort in applied mathematics to make progress in the development of low-dispersion and lowdissipation schemes for solving unsteady problems not only in fluid mechanics, but also in many other non-linear problems of physics.

Consider the following semi-discrete differential equation

$$
\frac{\partial u^{n}}{\partial t}=F\left(u^{n}, t\right)
$$

where $u^{n}(x)=u(x, n \Delta t)$. From the time Fourier transform defined as

$$
u(t)=\int_{-\infty}^{+\infty} \hat{u}(\omega) e^{-i \omega t} d \omega
$$

an amplification factor $R_{s}=\hat{u}^{n+1} / \hat{u}^{n}$ can be calculated. The integration error is estimated by comparison between the exact amplification factor given by $R e=e^{-i \omega \Delta t}$ 
and the effective amplification factor of the scheme, which can be written as follows [5]

$$
R_{S}=1+\sum_{j=1}^{p} \gamma_{j}(-i \omega \Delta t)^{j}
$$

Stability requires an amplification rate so that $\left|R_{s}(\omega \Delta t)\right|<1$, and integration errors can be measured by comparing $R_{s}=/ R_{s} / e^{-i \omega_{s} \Delta t}$ with the exact amplification factor $R_{e}$, in terms of dissipation error with $1-\mid R_{s} /$, and of phase error with $\left|\omega_{s} \Delta t-\omega \Delta t\right| / \pi$.

The amplification rates of some classical schemes are shown in figure 3 as a function of the normalized angular frequency $\omega \Delta t$. For frequency up to four points per wavelength, i.e. $\omega \Delta t<\pi / 2$, there is more than three orders of magnitude between the dissipation of the classical Runge-Kutta scheme and the optimized low-storage scheme of Berland et al., both providing a formal 4th-order integration. Note also the good behaviour of the optimized scheme for the phase error, and the large time-step range of stability, $\omega \Delta t<3.82$, with respect to the classical Runge-Kutta scheme yielding $\omega \Delta t<2 \sqrt{2} \simeq 2.83$.

Again this brief example is only reported to emphasize that new efficient algorithms have been developed over the last few years, with the aim of controlling numerical dispersion and dissipation for solving unsteady nonlinear problems.

\section{SUBSONIC JET NOISE}

The prediction of subsonic jet noise is one of the oldest topics of aeroacoustics $[26,27,37]$ even if our understanding of noise mechanisms remains incomplete. The final goal of all these research works is the reduction of noise in urban environments, and traffic growth must be compensated by innovative noise reduction methods. This environmental challenge is also strategic for the economic development of the aeronautics industry.

As pointed out in the introduction, the direct computation of aerodynamic noise using compressible large-eddy simulations is approaching maturity, and subsonic jet noise has been one of the first applications, with the direct numerical simulation of Freund [21] of a jet at Mach number 0.9 and at Reynolds number 3600, based on the jet exit velocity and the jet diameter. The grid requirement of direct numerical simulations is however difficulty to satisfy for the computations of laboratory experiments with typical Reynolds number $\operatorname{Re}_{D}$ of about $10^{5}-10^{6}$. Moreover, overall flow and noise characteristics are no longer dependent on the Reynolds number for roughly $R e_{D} \geq 2.5 \times 10^{5}$. This observation is directly linked to the laminar or turbulent state of the nozzle exit boundary layer [52]. Therefore, compressible large-eddy simulations appear to be relevant to develop direct noise computation and to reproduce Reynolds number effects.

To illustrate this point, figure 4 displays snapshots of the vorticity norm and of the fluctuating pressure for jets at Mach number 0.9 but at different Reynolds numbers in order to investigate alterations on the flow development and on the radiated acoustic field. In the present work, the LES strategy is based on explicit selective filtering with spectral-like resolution combined with low dispersion and low dissipation numerical algorithms, see reference $[5,53]$ for a discussion regarding the methodology. As the 

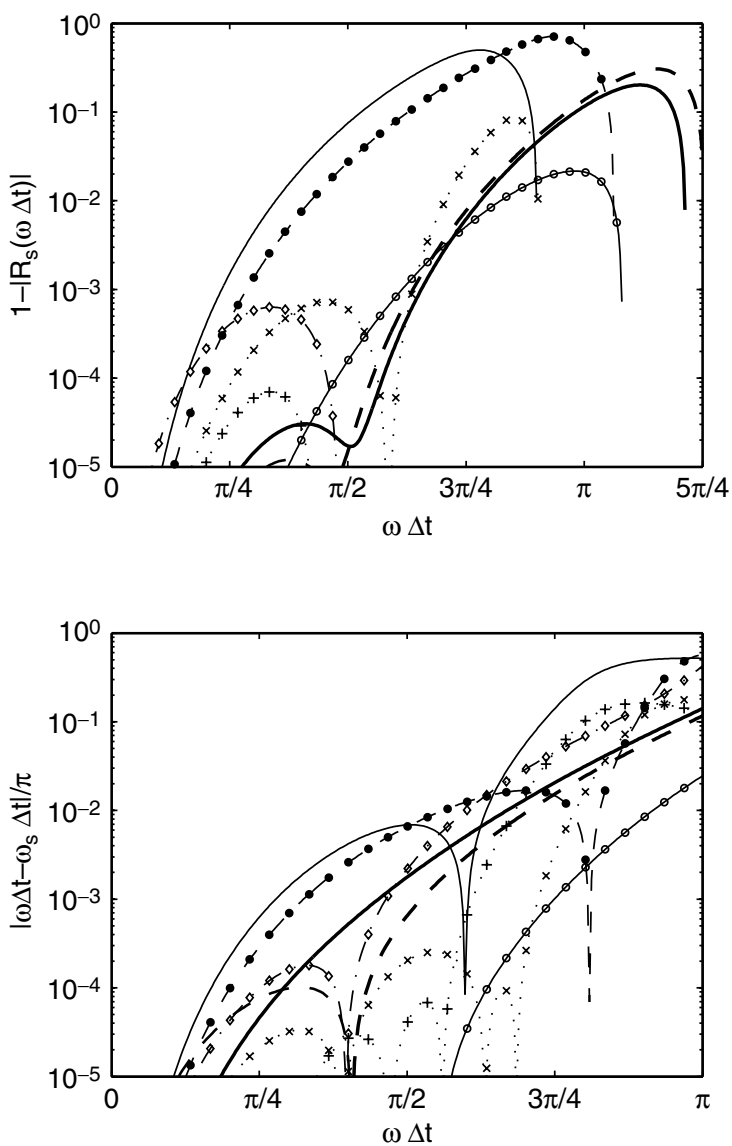

Figure 3: Modulus and phase error of the amplification factor (1) as a function of the angular frequency, plotted in logarithmic scale. standard 4th-order RK, - o - standard 8th-order RK, ․ + ‥ LDDRK46 Hu (1996), $\cdots \times \cdots$ LDDRK56 Hu (1996), - •- 4th-order 2N-RK Carpenter (1994), -• $\diamond$ opt. 4th-order 2N Stanescu (1998), - - - opt. 2nd-order RK Bogey (2004), — opt. 4th-order 2N-RK Berland (2006).

Reynolds number decreases, the jet flow changes significantly, and develops more slowly upstream of the end of the potential core, but more rapidly downstream. The acoustic field radiated in the sideline direction appears to vanish progressively as the Reynolds number is decreased, which can be directly linked to the absence of fine scale turbulence in the shear layers. Quantities such as mean velocity, jet spreading, turbulence intensity, integral length scales, spectra, acoustic azimuthal correlations and power laws have also been investigated as a function of the observer angle for circular jets at Mach number 0.6 and 0.9, with Reynolds numbers varying from $1.7 \times 10^{3}$ to $4 \times 10^{5}$ 

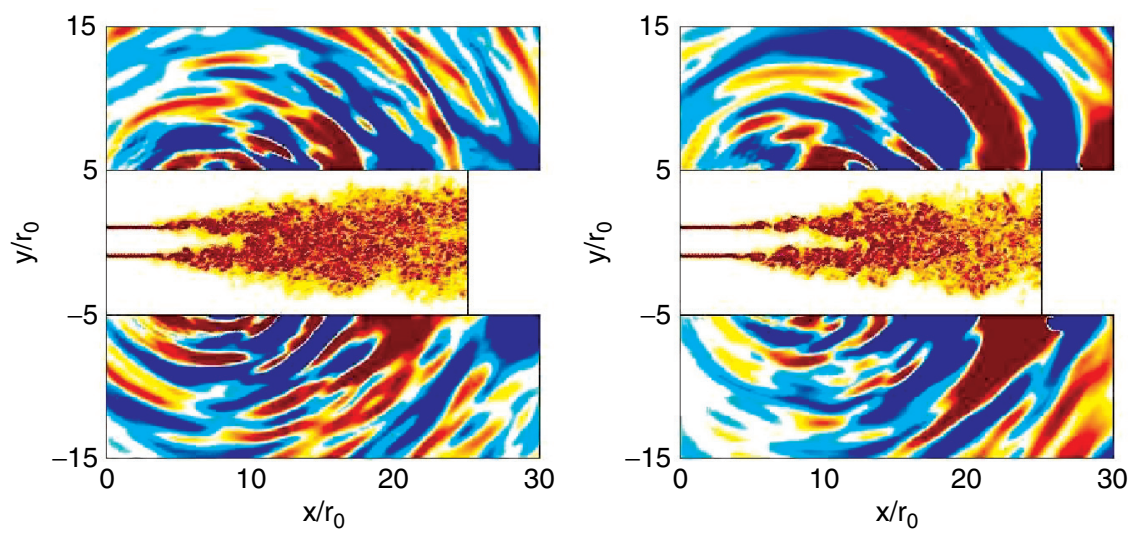

$\operatorname{Re}_{D}=4.5 \times 10^{5}$

$$
\mathrm{Re}_{D}=1.0 \times 10^{4}
$$

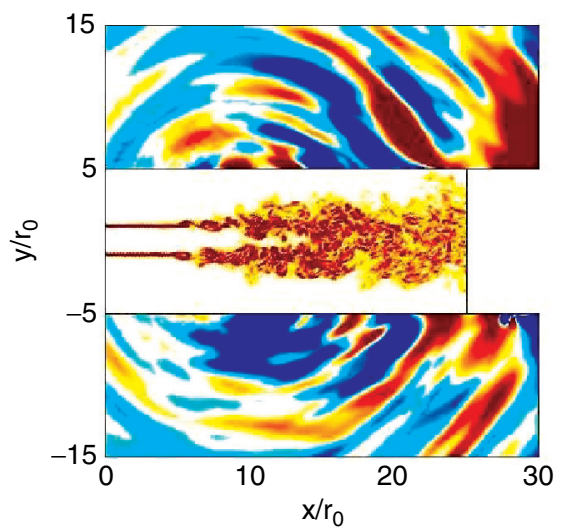

$\mathrm{Re}_{D}=5 \times 10^{3}$

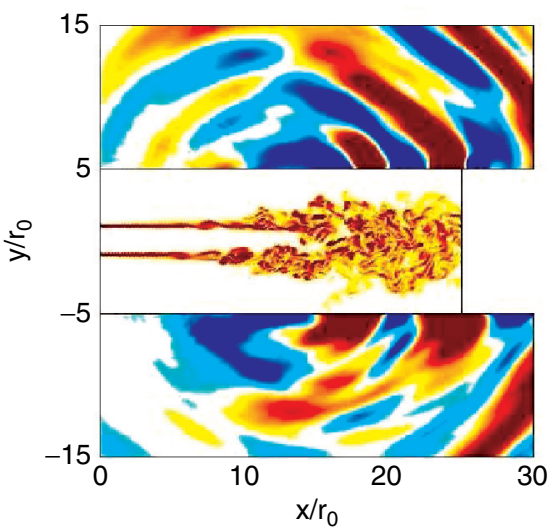

$\operatorname{Re}_{D}=2.5 \times 10^{3}$

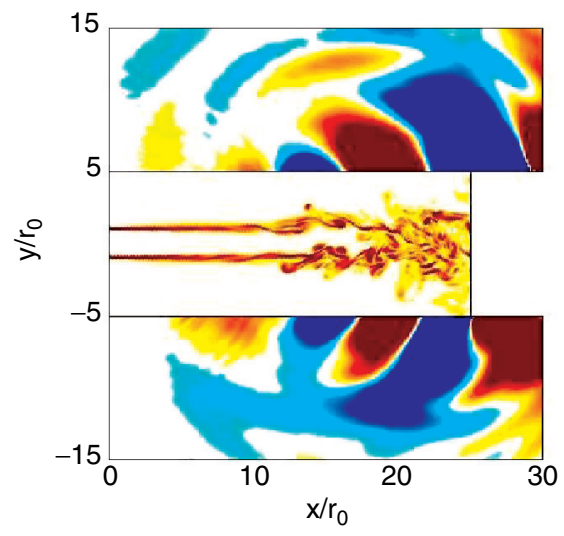

$\operatorname{Re}_{D}=1.7 \times 10^{3}$

Figure 4: Jets at Mach $M=0.9$. Snapshots of the vorticity norm $|\omega|$ in the flow and of the fluctuating pressure $p^{\prime}$ outside, in the plane $\mathrm{z}=0$. For the five simulations, the color scale of the vorticity norm is $|\omega| \times \mathrm{r}_{0} / u_{j}=[0,2.65]$, and the pressure color scale is $p^{\prime}=[-70,70] \mathrm{Pa}$ or $p^{\prime} /\left(\rho_{j} u_{j}^{2}\right)=[-6.2,6.2] \times 10^{-4}$. Taken in part from reference [8]. 
by Bogey and Bailly $[8,9]$. The simulations suggest the presence of two sound sources: a Reynolds-number-dependent source, predominant for large radiation angles, connected to the randomly-developing turbulence, and a deterministic source, radiating downstream, related to a mechanism intrinsic to the jet geometry, which is still to be comprehensively described. This view agrees well with the experimental results displaying two distinguishable components in turbulent mixing noise [47].

Furthermore for the acoustic spectra of the two apparent contributions, frequency scaling by a Strouhal number, $\mathrm{St}=f D / u_{j}, f$ being the frequency, $D$ the jet diameter and $u_{j}$ the jet velocity, appears suitable for both. However, the evolution of the peak is clearly different in the two directions, namely in the transverse direction and in the downstream direction. For observation angles $\theta \simeq 90 \mathrm{deg}$, the spectral peak is Strouhal-dependent, and must be connected to the turbulence development in the shear layers between the nozzle and the end of the potential core. This evolution is also clearly visible on spectra. In the downstream direction, the frequency is weakly dependent on the Reynolds number, with $\mathrm{St} \simeq 0.25$, and this radiation can be interpreted as a result of the periodic intrusion of vorticity at the end of the potential core.

The acoustic radiation by the turbulence developing in the shear layers seems partially understood, and active control or flow forcing by impinging microjets could be applied to achieve noise reduction. On the contrary, the noise mechanism at the end of the potential core is not well explained with our current knowledge of jet noise. Frequency selection of a global mode for subsonic cold jets is not predicted by the classical instability theory for instance, and is still to be clearly described. Based on this remark, it should be also underlined that there is still a role for theory, in particular to support the interpretation of these simulations.

Another possible way to establish direct links between turbulent flow events and emitted sound waves and to help towards the identification of noise-source mechanisms, is to apply a causality method to LES data, as proposed in Bogey and Bailly [10] for instance. For that, the normalized cross-correlation between the jet turbulence at $\left(x_{1} ; t_{0}\right)$ and the radiated pressure $\left(\boldsymbol{x}_{2} ; t_{0}+t\right)$ is introduced:

$$
C_{f_{p}}\left(\boldsymbol{x}_{1}, \boldsymbol{x}_{2}, t\right)=\frac{\left\langle f\left(\boldsymbol{x}_{1}, t_{0}\right) p^{\prime}\left(\boldsymbol{x}_{2}, t_{0}+t\right)\right\rangle}{\left\langle f^{2}\left(\boldsymbol{x}_{1}, t_{0}\right)\right\rangle^{1 / 2}\left\langle p^{\prime 2}\left(\boldsymbol{x}_{2}, t_{0}\right)\right\rangle^{1 / 2}}
$$

where the quantity $f$ is any relevant calculated variable of the direct noise computation. Results are reported in figure 5 where $f$ is the norm of the vorticity along the jet axis. The particular role played by the fluid dynamics at the end of the potential core is again emphasized for the noise radiated in the downstream direction whatever the Reynolds number may be. This kind of investigation clearly needs more work using advanced signal processing and alternative localization techniques such as antenna or conditional statistics.

To conclude and to provide a critical review, even if high-fidelity flow and noise simulations are now performed, it involves some difficulties such as the generation of artificial turbulence at the inflow boundary conditions to mimic the turbulent boundary layer or the thicker boundary layers used in numerical simulations, typically $\delta_{\theta} / D \sim 10^{-2}$ 

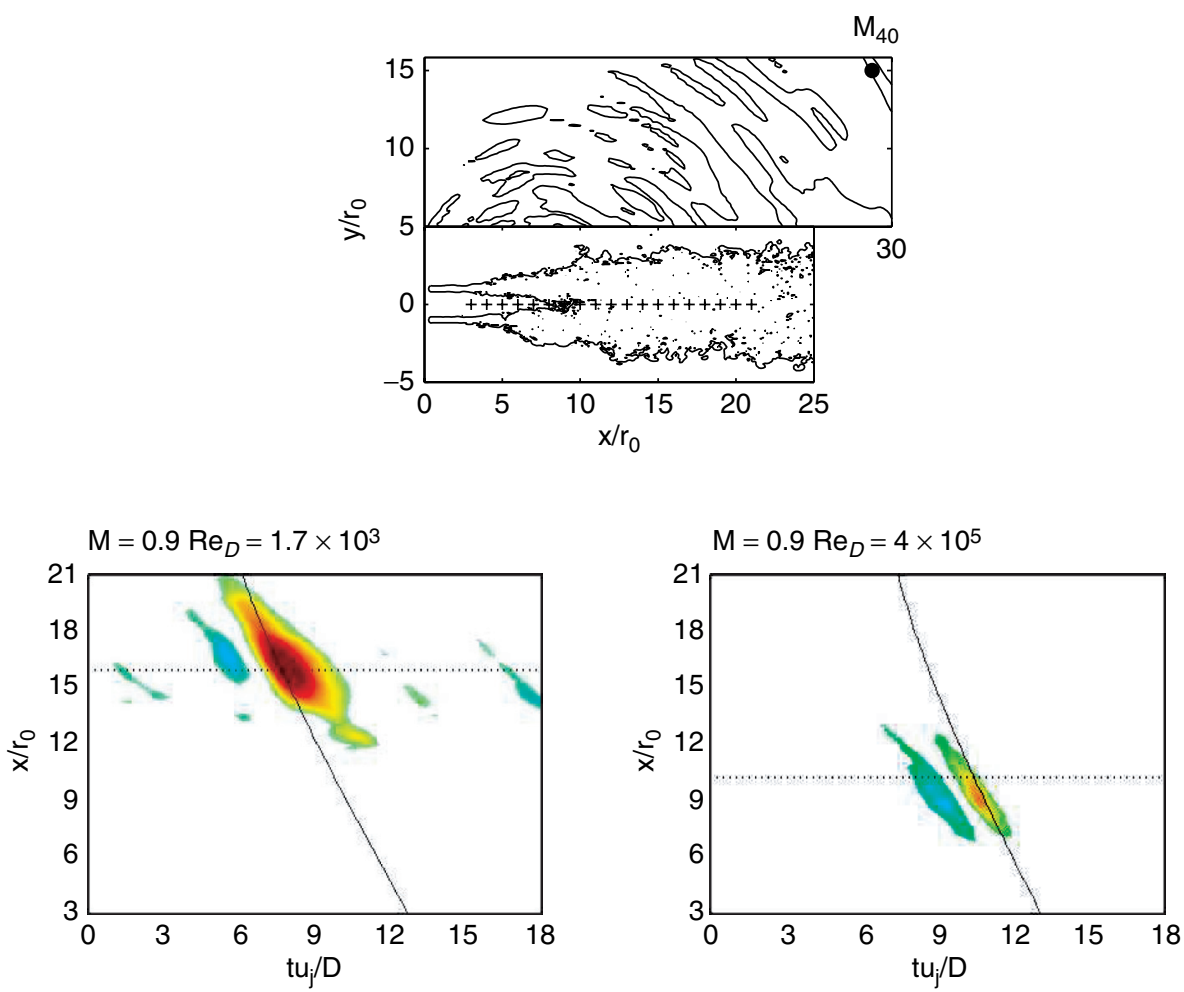

Figure 5: Correlation coefficient $\mathrm{C}_{\omega p}\left(x / r_{0} ; t u_{j} / D\right)$ between the vorticity along the jet axis at $+\boldsymbol{x}_{1}=(x, 0,0)$ and the acoustic field at $\bullet \boldsymbol{x}_{2}=M_{40}$. Time delay $t u_{j} / D$ versus axial distance $x / r_{0}$, time of propagation at the speed of sound,----- end of the potential core $x=x_{c}$, color scale: $\left[\begin{array}{lll}-0.14 & 0.14\end{array}\right]$ (white: [-0.035 0.035]). Taken from reference [10].

instead of $10^{-3}$ in experiments, leading to some potential shifts with measurements for the potential core length or spectral peaks in the initial shear layer [54].

\section{SUPERSONIC JET NOISE}

Additional noise generated by supersonic jets, and especially screech tones, contribute significantly to acoustic fatigue of combat aircraft. Shock-associated noise radiates primarily in the upstream direction and consequently increases also notably cabin noise of modern commercial aircrafts.

Noise of imperfectly expanded supersonic jets has been studied experimentally and theoretically in order to identify the interactions between turbulence and the quasiperiodic shock-cell structure. These interactions generate upstream-propagating sound waves. A resonant loop is then obtained when acoustic waves are diffracted by the 
nozzle lips and thus excite the initial shear layers. However, predictions are still qualitative and provide basically the fundamental frequency associated with the feedback loop. Further details can be found in the review paper of Raman [39]. The determination of the amplitude of the radiated acoustic field remains a difficulty challenge. The decrease of screech tone amplitude observed for heated jets or the influence of the nozzle-lip thickness have been numerically investigated by Shen and Tam [41] for round jets. Suzuki and Lele [43] have proposed an interpretation of the screech generation mechanism through a shock-leakage phenomenon.

This issue has been recently investigated by Berland et al. [6] with the compressible large eddy simulation of screech tones generated by a threedimensional planar underexpanded jet. The jet operates at fully expanded Mach number $\mathrm{M}_{j}=1.55$, with a Reynolds number $\mathrm{Re}_{h}=6 \times 10^{4}$ based on the jet exit velocity $u_{j}$ and of the nozzle height $h$. The ratio between the exit pressure and the ambient pressure is $p_{e} / p_{\infty}=2.09$, corresponding to maximum screech noise generated by a rectangular nozzle with large aspect ratio, as shown experimentally by Krothapalli et al. [25]. Numerical parameters of the simulation and validations can be found in [6]. The flow and especially the shock-cell structure are in agreement with the literature. Furthermore the upstream acoustic field exhibits harmonic tones that compare correctly to screech tones observed in rectangular jets in terms of frequency, amplitude and phase shift on both sides of the jet. As an illustration, figure 6 displays a snapshot of the direct noise computation. Compression shocks corresponding to high-density gradients are seen inside the jet plume. Upstreampropagating wave fronts associated with screech tones radiation are also clearly visible on both sides of the jet. The Strouhal number corresponding to the screech frequency is equal to $\mathrm{St}=f_{s} h / u_{j} \simeq 0.126$. A further study of the simulation data has allowed the location of the screech source near the third shock-cell, as noticed in the experiments of Krothapalli et al. among others, and to provide evidences of the connection between the shock-leakage process, proposed by Suzuki and Lele [43], and the generation of screech tones.

The far-field noise is extrapolated by using the linearized Euler equations in order to compute acoustic spectra. Power spectral densities of the pressure fluctuations are reported in figure 7 for different observation angles $\theta$ with respect to the downstream direction. Three contributions can be found: screech noise, broadband shockassociated noise and mixing noise which has already been discussed in the previous section devoted to subsonic jet noise. For $\theta=155 \mathrm{deg}$, the spectrum is dominated by the fundamental screech tone and its harmonics. For an observer in the sideline direction, $\theta=80 \mathrm{deg}$, the fundamental screech tone is no longer visible whereas its first harmonic dominates the radiated field. Two broadband peaks can also be noticed, a low-frequency contribution at $\mathrm{St} \simeq 0.07$ associated with the mixing noise and a higher frequency contribution around $0.1 \leq \mathrm{St} \leq 0.2$. In the downstream direction, at $\theta=40$ $\mathrm{deg}$, the mixing noise becomes the principal noise source. As pointed out by Tam et al. [46], a relationship can be derived between the frequency peak $f_{p}$ of broadband shockassociated noise 


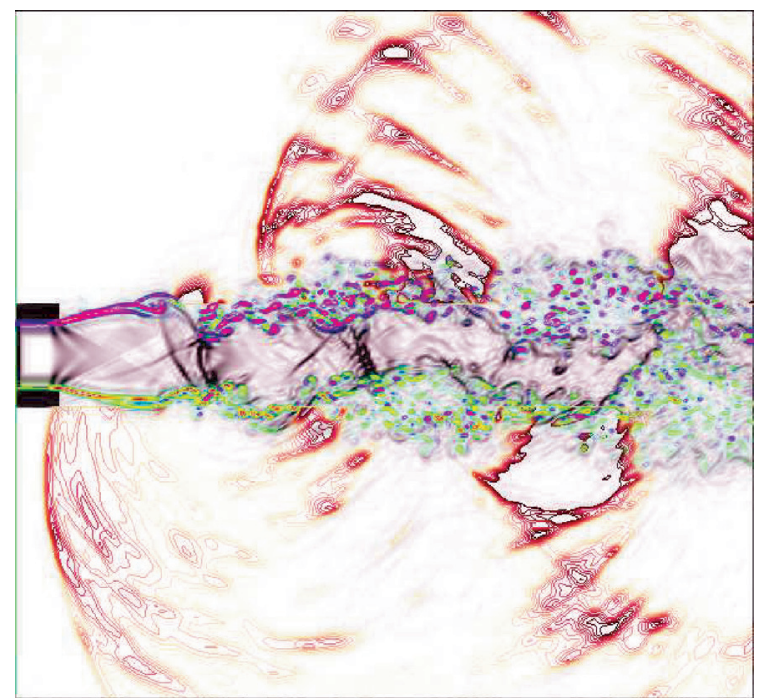

Figure 6: Computation of the generation of screech tones in an underexpanded supersonic jet, fully expanded jet at Mach number 1.55, Reynolds number $6 \times 10^{4}$, see reference [6] for details. Snapshot of the density modulus, of the spanwise vorticity and of the near-field pressure, in a plane perpendicular to the spanwise direction. The nozzle lips are represented in black.

$$
\frac{f_{p} h}{c_{\infty}} \simeq M_{c} \frac{h}{L_{s}\left(1-M_{c} \cos \theta\right)}
$$

and those of screech noise $f_{s}=\lim _{\theta \rightarrow \pi} f_{p}$, where $M_{c}=u_{c} / c_{\infty} \simeq 0.55 u_{j} / c_{\infty}$ is the convection Mach number and $L_{s}$ is the shock cell spacing, approximated by $L_{s} \simeq 2 h\left(M_{j}^{2}-1\right)^{1 / 2}$ for a two-dimensional jet, see Tam [44].

The present simulation is thus able to capture the three noise sources and to correctly reproduce broadband spectra as a function of the observer position, in agreement with the literature, as summarized by Tam [45] or by Raman [39].

\section{SELF-EXCITATED OSCILLATIONS IN INTERNAL FLOWS}

Shock-induced flow oscillations behind a sudden enlargement of cross-section are frequently generated by pressure-reducing valves and by flow control devices in pipe systems of power plants. This configuration is also representative of transonic and supersonic flows involving unstable shock patterns yielding noise production increase, as discussed in the survey paper of Meier et al. [31]

The present studied configuration, displayed in figure 8 , has been investigated experimentally for different values of the area ratio $h / H$ and of the channel length $L / H$. 


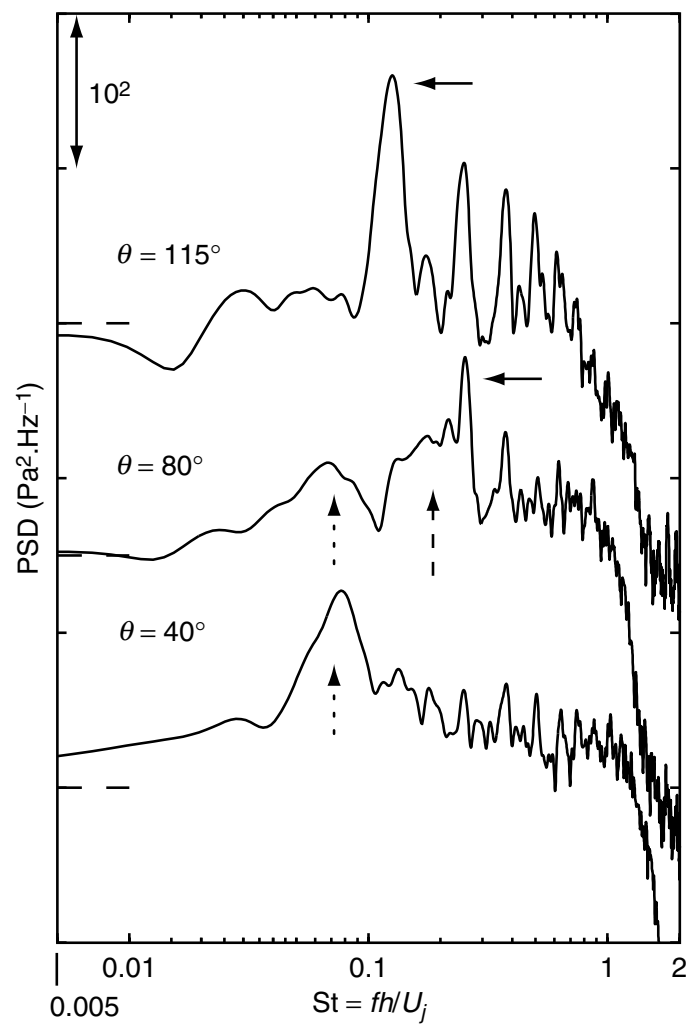

Figure 7: Generation of screech tones in an underexpanded supersonic jet [6], fully expanded at jet Mach number 1.55, Reynolds number $6 \times 10^{4}$. Computed pressure spectra in the acoustic far-field for three angles $\theta$ with respect to the downstream direction. The solid arrows stand for fundamental $f_{s}$ and first harmonic $2 f_{s}$ screech tones, the dashed arrow stands for broadband shock associated noise and the dotted arrows stand for mixing noise.

Boundary layer - shock wave interactions as well as possible flow oscillations, hysteresis phenomena and coupling with acoustic duct resonance are reported by Anderson et al. [1] and by Meier et al. [30]

The evolution of the mean base pressure $p_{w} / p_{a}$ as a function of the pressure ratio $\tau$ is shown in figure 9 for a given duct geometry. For lower values of the pressure ratio, $\tau \leq 0.25$, the mean base pressure $p_{w}$ is nearly constant. The symmetrical and steady flow pattern consists of reflected oblique shock waves interacting with the boundary layers. Increasing the plenum-chamber pressure $p_{e}$, and thus the pressure ratio $\tau=p_{e} / p_{a}$ where $p_{a}$ is the reservoir pressure, leads to a decrease and at the end, to a breakdown of the 


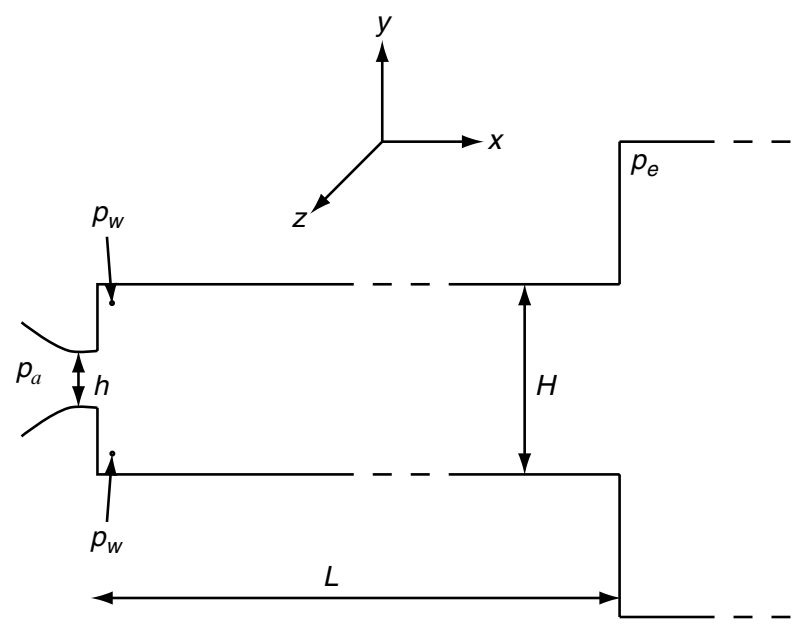

Figure 8: Sudden enlargement of rectangular duct cross-section: the flow regime is characterized by the pressure ratio between the plenum chamber and the reservoir pressure $\tau=p_{e} / p_{a}$. The area ratio is given by $h / H$, where $h$ is the nozzle height and $H$ the height of the test-duct; $L$ is the duct length and $b$ is the width of the nozzle and of the duct. The flow can be monitored by the base pressure $p_{w}$ at the two upstream corners of the channel.

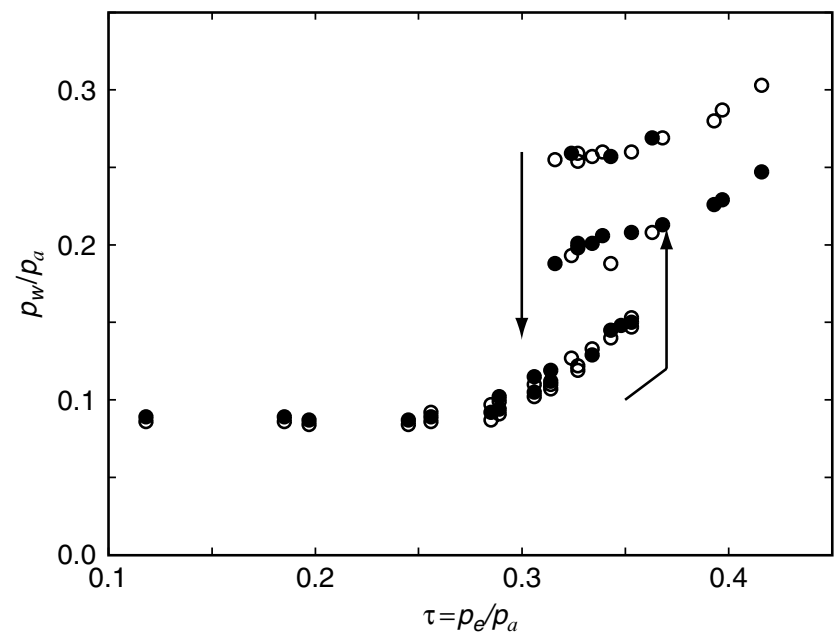

Figure 9: Turbulent flow behind a sudden enlargement of rectangular duct crosssection for the case $L=0.16 \mathrm{~m}, L / H=5.23$ and $h / H=0.3$. Experimental data of the upper $\circ$ and lower $\bullet$ normalized mean base pressure $p_{w} / p_{a}$ as a function of the pressure ratio $\tau$, data from Anderson et al. [1] 
(a) $\tau=0.31$

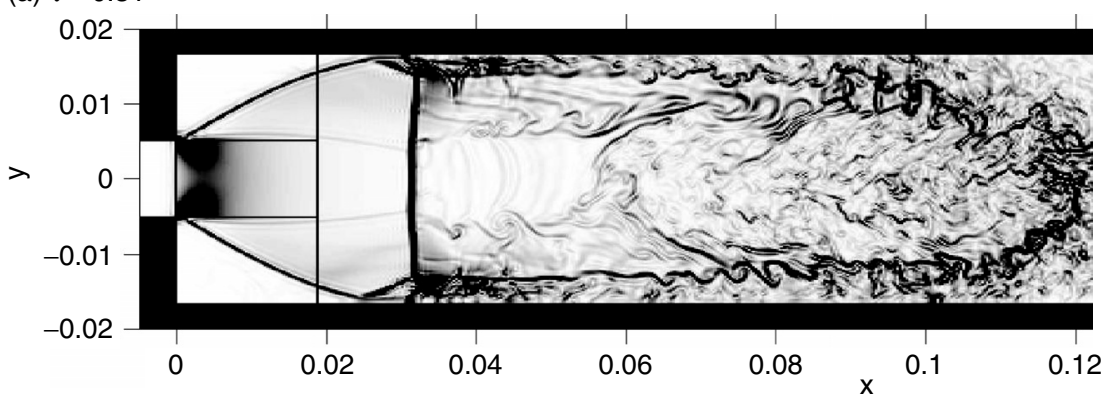

(b) $\tau=0.32$

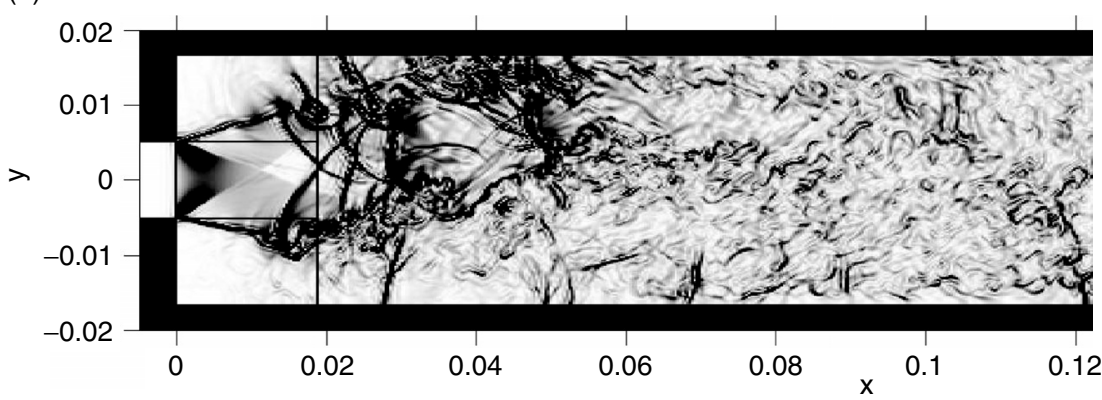

Figure 10: Compressible large-eddy simulation of the turbulent flow behind a sudden enlargement of rectangular duct cross-section: snapshot of density gradient $/ \nabla \rho$ / for two pressure ratios for the case $L=0.16 \mathrm{~m}, L / H=5.23$ and $h / H=0.3$. See Emmert et al. [19] for details.

periodic shock-cell structure. For higher pressure ratio, the flow becomes asymmetric and is attached to either the upper or the lower wall of the channel yielding two values of the base pressure $p_{w}$. An hysteresis phenomenon is also observed experimentally, indicated by the two arrows in figure 9. Moreover a self-excited flow configuration can occur for $0.31 \leq \tau \leq 0.35$, and under particular conditions, a strong coupling is found with longitudinal acoustic modes of the channel, at frequencies given by

$$
f=(2 n-1) \frac{c}{4 L}\left(1-\bar{M}^{2}\right) \quad n=1,2, \ldots
$$

where $\bar{M}$ is the averaged Mach number along the channel axis.

This flow has been carefully studied in Emmert et al. [19] by compressible largeeddy simulations based on a high-order algorithm and an additional non-linear adaptive filtering combined with high-order overlapping grid technique. Schlieren pictures of two computed regimes among others, are displayed in figure 10 . For $\tau=0.31$, a symmetrical flow pattern is found with a normal shock wave in the channel. A 
characteristic lambda structure for the shock foot is observed as well as interactions with wall boundary layers producing their thickening behind the shock. In the case $\tau=0.32$, an asymmetrical separated flow is found with attachement to the upper side of the channel. The switching between the symmetrical and asymmetrical flow patterns as well as the predicted values for the base pressure have been accurately reproduced by the simulations. A case of strong coupling between shock oscillations and a longitudinal acoustic mode is also reported by Emmert et al. [19]

The simulation of such configurations involving turbulence, boundary layers, shocks and coupling through acoustic resonances is currently challenging. The hysteresis cycle described in figure 9 and the symmetric or asymmetrical state taken by the shock pattern is strongly dependent on the correct description of the turbulent boundary layers as well as of the impedance conditions at the boundaries of the computational domain. A suitable truncation of the physical domain is also of importance.

\section{SIMULATION OF THE APPLICATION OF AN ADAPTIVE CONTROL TO A CAVITY FLOW}

Cavity noise, which occurs when a cavity is placed in a grazing flow, is of increasing concern to both military applications for the flow inside the cavity, and to the transport industry for the radiated far-field noise. Instabilities are shed by the upstream cavity corner and are simultaneously convected and amplified by the shear layer until they impact the downstream cavity wall, thus generating noise. Pressure waves induced by the impact can create a feedback loop by synchronising the upstream shear layer oscillations, resulting in very high pressure fluctuation levels. Active control techniques have been investigated experimentally as possible means of reducing noise generation. Recent syntheses of the extensive litterature can be found in Cattafesta et al. [13] or in Rowley and Williams [40].

A direct simulation of a closed-loop active control to reduce cavity noise is reported in this section. A leaky-Least Mean Square (LMS) algorithm has been used and implemented in the compressible Navier-Stokes solver developed by Gloerfelt et al. [23] to show the feasability of the control itself, and of the numerical coupling. The simulated cavity is shown in figure 11 . The cavity is $2 \mathrm{~mm}$ long, with $L / D=1$ and $L / W=1.28$. The upstream flow has a Mach number of $M=0.6$ and a Reynolds number based on the cavity depth of $R e_{D}=28720$. The upstream boundary layer is laminar, with $L / \delta_{\theta}=57$. A simple form of pulsed injection is used as the control actuator. It is accomplished by adding a control term to the momentum equation on $\rho v$ where $v$ is the vertical velocity, inside the time integration. This term is introduced in a zone whose envelope is Gaussian in the $x$ and $y$ directions and of half-width $L / 50$ in both directions. The injection zone, spanning the entire width of the cavity, is placed immediately after the upstream corner, as shown in figure 11. The error signal supplied to the control algorithm is the pressure perturbation $p^{\prime}=p-p_{\infty}$ measured slightly underneath the impact zone on the downstream cavity wall and averaged over five sensors in the spanwise direction.

The stability of the controlled cavity is one of the more delicate aspects of the simulation. It was found that the total elimination of upstream instabilities, although 


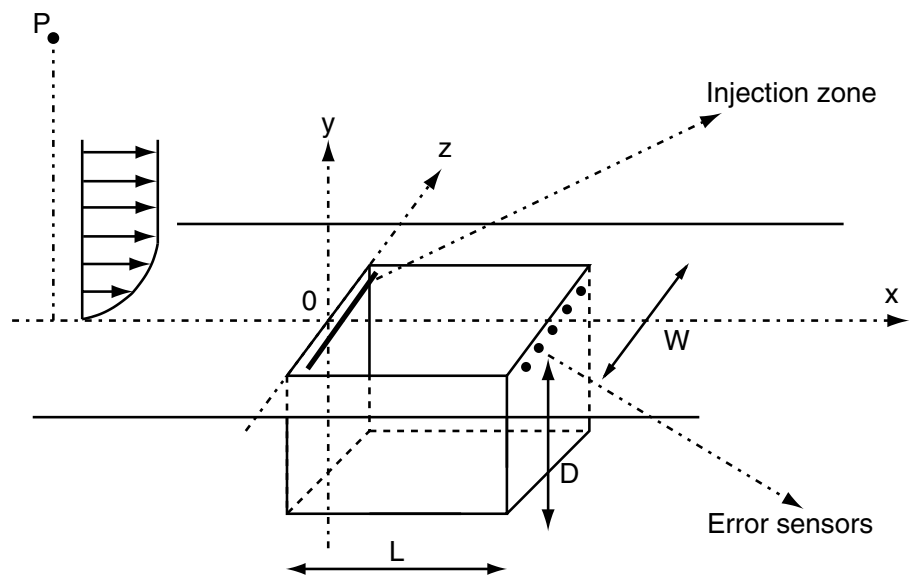

Figure11: Sketch of the simulated cavity layout [28].

possible for a short time, must be avoided in order to obtain a stable state. Indeed if the upstream instabilities become too small, they can very easily be perturbed and end up in phase with the injected control signal, leading to positive feedback and rapid divergence. The control algorithm should therefore not converge to an error value of zero, since this state is unstable. Rather than impose a non-zero sinusoidal error toward which to converge, a leaky-LMS approach with a strong leakage factor was used, to avoid the error signal becoming too small. This method both avoids having to establish a priori a target error signal to obtain, and also increases the algorithm's response speed to phase and frequency changes in the error signal. Figure 12 displays the signal pressure measured at point $P$, and illustrates the noise reduction when the control system is started. Analysis of the flow, the sound field and details about implementation of the feedback loop are provided in Marsden et al. [28]

\section{WORK IN PROGRESS}

Among different topics that can be mentioned as work in progress, we can highlight the increasing complexity in physics, in numerics and in studied geometries. The first category includes large-eddy simulations involving high-Reynolds number flows, heated flows, or transonic and supersonic flows [11] for which the accuracy is difficult to retain. Turbulence modelling itself remains a key issue that is still unresolved, at least for DNC, and needs to be objectively examined with the knowledge of the transfer function of the numerical algorithm, as suggested by Domaradzki and Adams [18] or more recently by Berland et al. [7] for optimized finite-difference schemes. The methodology to specify the inflow boundary conditions of turbulent boundary layers in the framework of DNC likewise remains a challenging task, the reader may refer to $\mathrm{Xu}$ and Martin [51] for a recent discussion. Finally, simulation of more complex geometries involving couplings with structure in aeroelasticity, are also of importance and can be tackled by the use of 


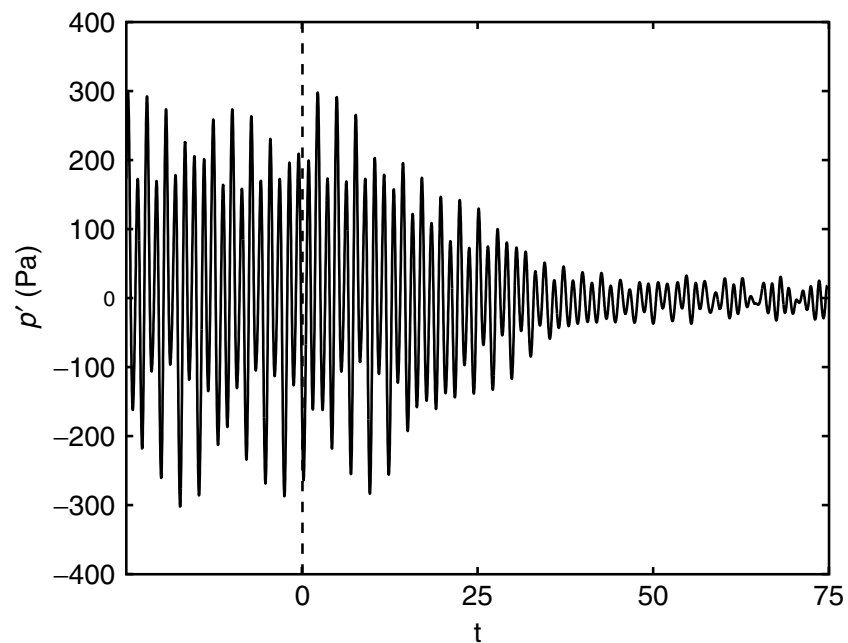

Figure12: Numerical simulation of a closed-loop active control in a 3-D cavity. Time evolution of the pressure radiated at point $P$, refer to Figure 11, before and after control. Control phase starts at $t=0$. From Marsden et al. [28]

high-quality block structured grids. Several research teams have developed such techniques with the aim of aeroacoustic [42] and aeroelastic applications [49]. Efforts are also now underway to develop unstructured approaches [48] for realistic applications, even if accuracy and robustness seem still difficult and costly to preserve.

\section{ACKNOWLEDGMENTS}

A preliminar version was presented at the 7th International ERCOFTAC Symposium on Engineering Turbulence Modelling and Measurements (ETMM7), and the first author thanks the organizers for their invitation and their financial support. The authors would like also to thank Julien Berland and Xavier Gloerfelt from SINUMEF, ENSAM Paris, Thomas Emmert and Philippe Lafon from Electricité de France \& LaMSID, UMR CNRS 2832, and Mélanie Piellard from Delphi Thermal Systems, Luxembourg, for the sharing of their results. The authors gratefully acknowledge the Institut du Développement et des Ressources en Informatique Scientifique (IDRIS) of the CNRS and the Centre de Calcul Recherche et Technologie of the CEA (the French Atomic Energy Agency) for providing CPU time on Nec computers and technical assistance. The first author is also with Institut Universitaire de France.

\section{REFERENCES}

[1] Anderson, J. S., Jungowski, W. M., Hiller, W. J. \& Meier, G. E. A., "Flow oscillations in a duct with a rectangular cross-section", J. Fluid Mech., Vol. 79, No. 4, 1977, pp. 769-784. 
[2] Bailly, C., Lafon, P. \& Candel, S., "Prediction of supersonic jet noise from a statistical acoustic model and a compressible turbulence closure", J. Sound Vib., Vol. 194, No. 2, 1996, pp. 219-242.

[3] Bailly, C., Lafon, P. \& Candel, S., "Subsonic and supersonic jet noise predictions from statistical source models", AIAA Journal, Vol. 35, No. 11, 1997, pp. 1688-1696.

[4] Bailly, C. \& Bogey, C., "Contributions of computational aeroacoustics to jet noise research and prediction", International Journal of Computational Fluid Dynamics, Vol. 18, No. 6, 2004, pp. 481-491.

[5] Berland, J., Bogey, C. and Bailly, C., "Low-dissipation and low-dispersion fourthorder Runge-Kutta algorithm”, Computer \& Fluids, Vol. 35, No. 10, 2006, pp. 1459-1463.

[6] Berland, J., Bogey, C. and Bailly, C., "Numerical study of screech generation in a planar supersonic jet", Phys. Fluids, Vol. 19, 075105, 2007, pp. 1-14.

[7] Berland, J., Bogey, C. and Bailly, C., "Investigation using statistical closure theory of the influence of the filter shape on scale separation in large-eddysimulation", Journal Of Turbulence, http://jot.iop.org/, Vol. 9, No. 21, 2008, pp. 1-22.

[8] Bogey, C. and Bailly, C., "Investigation of downstream and sideline subsonic jet noise using Large Eddy Simulations", Theoretical and Computational Fluid Dynamics, Vol. 20, No. 1, 2006, pp. 23-40.

[9] Bogey, C. and Bailly, C., "Large Eddy Simulations of transitional round jets: influence of the Reynolds number on flow development and energy dissipation", Physics of Fluids, Vol. 18, 065101, 2006, pp. 1-14.

[10] Bogey, C. and Bailly, C., "An analysis of the correlations between the turbulent flow and the sound pressure field of subsonic jets", Journal of Fluid Mechanics, Vol. 583, 2007, pp. 71-97.

[11] Bogey, C., De Cacqueray, N. and Bailly, C., "A shock-capturing methodology based on adaptative spatial filtering for high-order non-linear computations", Journal of Computational Physics, Vol. 228, No. 5, 2009, pp. 1447-1465.

[12] Bui, T. P., Schröder, W. \& Meinke, M., “Acoustic perturbation equations for reacting flows to compute combustion noise", Aeroacoustics, Vol. 6, No.4, 2007, pp. 335-355.

[13] Cattafesta III, L. N., Williams, D., Rowley, C. and Alvi, F., "Review of active control of flow-induced cavity resonance", AIAA Paper 2003-3567.

[14] Colonius, T. (2001), An overview of simulation, modeling and active control of flow/acoustic resonance in open cavities, AIAA Paper 2001-0076, pp. 1-12.

[15] Colonius, T., "Modeling artificial boundary conditions for compressible flows", Annu. Rev. Fluid Mech., Vol. 36, 2004, pp. 315-345.

[16] Colonius, T. and Lele, S., "Computational aeroacoustics: progress on nonlinear problems on sound generation", Progress in Aerospace Sciences, Vol. 40, 2004, pp. $345-416$. 
[17] Curle, N., "The influence of solid boundaries on aerodynamic sound", Proc. Roy. Soc. London, Vol. A231, No. 1187, 1955, pp. 505-514.

[18] Domaradzki, J. A. \& Adams, N. A, "Direct modelling of subgrid scales of turbulence in large eddy simulations", Journal Of Turbulence, http://jot.iop.org/, Vol. 3, No. 024, 2002, pp. 10-19.

[19] Emmert, T., Lafon, P. and Bailly, C., "Numerical study of self-induced transonic flow oscillations behind a sudden duct enlargement", Phys. Fluids, Vol. 21 , 2009.

[20] Ffowcs Williams, J. E. and Hawkings, D. L., Sound generation by turbulence and surfaces in arbitrary motion, Phil. Trans. Roy. Soc. London, Vol. 264, Ser. A, 1151, 1969 , pp. 321-342.

[21] Freund, J.B., "Noise sources in a low-Reynolds-number turbulent jet at Mach 0.9”, J. Fluid Mech., Vol. 438, 2001, pp. 277-305.

[22] Gloerfelt, X., Pérot, F., Bailly, C. and Juvé, D., "Flow-induced cylinder noise formulated as diffraction problem for low Mach number flows", J. Sound Vib., Vol. 287, No. 1-2, 2005, pp. 129-151.

[23] Gloerfelt, X., Bogey, C. and Bailly, C., "Numerical evidence of mode switching in the flow-induced oscillations by a cavity", International Journal of Aeroacoustics, Vol. 2, No. 2, 2003, pp. 193-217.

[24] Goldstein, M. E., “An exact form of Lilley's equation with a velocity quadrupole/temperature dipole source term", J. Fluid Mech., Vol. 443, 2001, pp. 231-236.

[25] Krothapalli, A., Hsia, Y., Baganoff, D. and Karamcheti, K., "The role of screech tones in mixing of an underexpanded rectangular jet", J. Sound Vib., Vol. 106, No. 1, 1986, pp. 119-143.

[26] Lighthill, M.J., "On sound generated aerodynamically - I. General theory”, Proc. Roy. Soc. London, Vol. 211, Ser. A, 1107, 1952, pp. 564-587.

[27] Lilley, G. M., "On the noise from air jets", British Aeronautical Research Council, A.R.C. 20-276, 1958.

[28] Marsden, O., Gloerfelt, X. and Bailly, C., "Direct noise computation of adaptive control applied to a cavity flow", C. R. Méc., Acad. Sci. Paris, Vol. 331, No. 6, 2003, pp. 423-429.

[29] Marsden, O., Bogey, C. and Bailly, C., "Direct noise computation of the turbulent flow around a zero-incidence airfoil", AIAA Journal, Vol. 46, No. 4, pp. 874-883.

[30] Meier, G.E.A., Grabitz, G., Jungowski, W.M., Witczak, K.J. and Anderson, J.S. (1978) Oscillations of the supersonic flow downstream of an abrupt increase in duct cross-section, Mitteilung aus dem Max-Planck-lnstitut fuer Stroemungsforschung und der Aerodynamischen Versuchsanstalt, Göttingen, Vol. 65.

[31] Meier, G.E.A., Szumowski, A.P. and Selerowicz, W.C., "Self-excited oscillations in internal transonic flows", Prog. Aerospace Sci., Vol. 27, 1990, pp. $145-200$. 
[32] Möhring, W., Müller, E.-A. \& Obermeier, F., "Problems in flow acoustics", Rev. Mod. Phys., Vol. 55, No. 3, 1983, pp. 707-724.

[33] Morris, P. J. \& Farassat, F., "Acoustic analogy and alternative theories for jet noise predictions", AIAA Journal, Vol. 40, No. 4, 2002, pp. 671-680.

[34] Oberai, A. A., Ronaldki, F. and Hughes, T. J. R. (2000), Computational procedures for determining structural-acoustic response due to hydrodynamic source, Comput. Methods Appl. Mech. Engrg., Vol. 190, pp. 345-361.

[35] M. Omais, B. Caruelle, S. Redonnet, E. Manoha, P. Sagaut, "Jet Noise Prediction Using RANS CFD Input", AlAA-Paper 2008-2938.

[36] Piellard, M., and Bailly, C. (2008), Validation of a hybrid CAA method and application to the case of a diaphragm in a duct at low Mach number, 14th AIAA/CEAS Aeroacoustics Conference, Paper 2008-2873.

[37] Powell, A., "The influence of the exit velocity profile on the noise of a jet", Aeronautical Quaterly, Vol. 4, 1953, pp. 341-360.

[38] Prax, C., Golanski, F. \& Nadal, N., "Control of the vorticity mode in the linearized Euler equations for hybrid aeroacoustic prediction", J. Comput. Phys., Vol. 277, 2008, pp. 6044-6057.

[39] Raman, G., "Supersonic jet screech: half-century from Powell to the present", J. Sound Vib., Vol. 225, No. 3, 1999, pp. 543-571.

[40] Rowley, C.W. and Williams, D.R., "Dynamics and control of high-Reynoldsnumber flow over open cavities", Аnnu. Rev. Fluid Mech., Vol. 38, 2006, pp. 251-276.

[41] Shen, H. and Tam, C. K. W., "Effects of jet temperature and nozzle-lip thickness on screech tones", AIAA Journal, Vol. 38, No. 5, 2000, pp. 762-767.

[42] Sherer, S. E. and Scott, J. N., "High-order compact finite-difference methods on general overset grids", J. Comput. Phys., Vol. 210, 2005, pp. 459-496.

[43] Suzuki, T. and Lele, S., "Shock leakage through an unsteady vortex-laden mixing layer: application to screech jet", Journal Fluid Mech., Vol. 490, 2003, pp. 139-167.

[44] Tam, C.K.W., "The shock-cell structures and screech tone frequencies of rectangular and non-axisymmetric supersonic jets", J. Sound Vib., Vol. 121, No. 1, 1988, pp. 135-147.

[45] Tam, C.K.W. (1995) Supersonic jet noise, Annu. Rev. Fluid Mech., Vol. 27, pp. $17-43$.

[46] Tam, C.K.W., Seiner, J.M. and Yu, J.C., "Proposed relationship between broadband shock associated noise and screech tones", J. Sound Vib., Vol. 110, No. 2, 1986, pp. 309-321.

[47] Tam, C. K. W., Viswanathan, K., Ahuja, K. K. and Panda, J., "The sources of jet noise: experimental evidence”, J. Fluid Mech., Vol. 615, 2008, pp. 253-292.

[48] Utzmann, J., Schwartzkopff, T., Dumbser, M. \& Munz, C.-D., "Heterogeneous domain decomposition for computational aeroacoustics", AIAA Journal, Vol. 44, No. 10, 2006, pp. 2231-2250. 
[49] Visbal, M. R. \& Gordnier, R. E., 2004, "Numerical simulation of the interaction of a transitional boundary layer with a 2-D flexible panel in the subsonic regime", Journal Fluids Struct., Vol. 19, No. 7, 2004, pp. 881-903.

[50] Wang, M., Freund, J.B. and Lele, S.K., "Computational prediction of flowgenerated sound", Annu. Rev. Fluid Mech., Vol. 38, 2006, pp. 483-512.

[51] Xu, S. and Martin, M. P., "Assessment of inflow boundary conditions for compressible turbulent boundary layers", Phys. Fluids, Vol. 16, No. 7, 2004, pp. 2623-2639.

[52] Zaman, K. B. M. Q., "Effect of initial condition on subsonic jet noise", AIAA Journal, Vol. 23, No. 9, 1985, pp. 1370-1373.

[53] Bogey, C. and Bailly, C., "Turbulence and energy budget in a self-preserving round jet: direct evaluation using large-eddy simulation", J. Fluid Mech., Vol. 627, 2009, pp. 129-160

[54] Bogey, C. and Bailly, C., "Influence of the nozzle-exit boundary-layer thickness on the flow and acoustic fields of initially laminar jets", 15th AIAA/CEAS Aeroacoustics Conference, AIAA Paper 2009-3409. 
